\title{
Recent Results on SUSY and Exotica Searches at the LHC
}

\author{
Zara Grout ${ }^{1, a}$ \\ ${ }^{1}$ University of Sussex, United Kingdom
}

\begin{abstract}
A review is presented of searches for Beyond the Standard Model (BSM) physics at the ATLAS and CMS experiments at the Large Hadron Collider (LHC) with emphasis on most recent results. This includes a summary of Supersymmetry searches covering strong, electroweak, and R-parity violating scenarios, and a range of exotica searches including heavy boson searches, extra dimensions, and long-lived particles. The status of BSM physics after the first data-taking period (Run 1) of the LHC and the prospects for the second data-taking period (Run 2), beginning in 2015, are discussed.
\end{abstract}

\section{Introduction}

The Standard Model (SM) has enjoyed astounding experimental success over the past few decades, most recently with the discovery of a Higgs Boson [1], [2]. However, unexplained phenomena and problems, for example dark matter, the difficulty of incorporating gravity, and the hierarchy problem [6], suggest that beyond the Standard Model (BSM) physics are required to accurately describe the universe we observe. Many such new physics models exist, conventionally separated into supersymmetry models, and all other BSM models, referred to as exotica. This provides a rich terrain to probe experimentally, and both ATLAS [4] and CMS [5] experiments at the Large Hadron Collider (LHC) [3] have run extensive physics programs to cover as many scenarios as possible. These proceedings will review results from some of the many analyses using the LHC Run 1 dataset from 2012, with $L=20.3 \mathrm{fb}^{-1}$ of proton-proton(pp) data recorded by ATLAS and $L=19.5 \mathrm{fb}^{-1}$ recorded by CMS, at a centre of mass energy of $\sqrt{s}=8 \mathrm{TeV}$. The selected analyses represent those which have become public most recently, and a variety of searches from ATLAS and CMS have been presented in order to demonstrate the diversity of BSM work, rather than due to differences in signatures or searches covered by the two experiments.

\section{Supersymmetry}

Supersymmetry (SUSY) [7] provides a resolution to the hierarchy problem [6], which refers to divergent corrections to the Higgs mass squared parameter due to loop diagrams of couplings between the Higgs and massive SM particles. A symmetry relating fermions to bosons is proposed, resulting in a supersymmetric partner for each SM particle with spin differing by $\frac{1}{2}$. All SM fermions will

\footnotetext{
ae-mail: zgrout@cern.ch
}

have a SUSY boson partner, and all SM bosons will have a SUSY fermion partner. These SUSY partners are at a displaced mass scale due to the breaking of supersymmetry, the exact nature of which is not fixed, except that it must be soft (i.e. added terms must be gauge invariant) in order to maintain the desirable features of SUSY.

Returning to the divergent Higgs mass squared correction terms, there is now a cancellation between the divergent loop corrections of the SM particles, and the equivalent terms from their supersymmetric partners. Models with lights SUSY partners of the heavier SM particles e.g. a light stop, are referred to as more "natural", as the cancellations will be closer to zero.

In addition to solving the Hierarchy problem, SUSY also leads to the unification of the strong, weak, and electromagnetic forces, and many scenarios provide a suitable dark matter candidate. This has caused it to be considered one of the most popular BSM theories, and both ATLAS and CMS have an extensive range of analyses covering many of the possible phenomenologies resulting from many additional parameters which are not strongly constrained. We will first discuss the search strategy generally employed by SUSY search analyses, before moving on to results from a selection of recent analyses. These will be separated depending on the production mode, which differ in production cross-section at the LHC and the most likely decay products.

\subsection{R-parity}

A new quantum number can be defined, which will differ for SM (+1) and SUSY (-1) particles. Referred to as "Rparity", it is defined as:

$$
R_{P}=(-1)^{3(B-L)+2 S},
$$

where, B, L and $\mathrm{S}$ are the baryon number, lepton number, and spin of the particle in question. As SUSY and SM par- 
ticles have different values, conserving this quantum number forbids the decay of the lightest SUSY particle. This is assumed for all models unless stated otherwise, however, it is possible to consider scenarios where this is not the case. We can include additional terms in our "superpotential" - describing the supersymmetric theory - which violate either baryon number or lepton number:

$$
W_{R P V}=\lambda_{i j k} L_{i} L_{j} \bar{E}_{k}+\lambda_{i j k}^{\prime} L_{i} Q_{j} \bar{D}_{k}+\mu_{i}^{\prime} L_{i} H_{2}+\lambda_{i j k}^{\prime \prime} \bar{U}_{i} \bar{D}_{j} \bar{D}_{k} .
$$

The indices i, j, k refer to lepton flavour, $L, E, Q, U, D$ and $H$ are superfields, and $\lambda, \lambda^{\prime}, \lambda^{\prime \prime}$ and $\mu^{\prime}$ are couplings [7]. The first three terms violate lepton number, and the final term violate baryon number. They all cause R-parity conservation to be violated. Due to lower limits of the proton lifetime of $10^{30}$ years, we cannot violate both lepton and baryon number, as this would facilitate proton decay. So the coupling for one interaction is chosen to be non-zero, and the other terms are ignored. This still results in an R-parity violating (RPV) scenario, leading to a decaying LSP. We will discuss results for models which consider $\mathrm{R}$-parity violation in a separate section.

\section{Search Strategy}

Analyses searching for SUSY typically employ a reasonably similar strategy, which initially involves identifying some signature of interest - expected from a SUSY model. "Simplified models" are often used to represent a signal, where only a few SUSY particles are set with light enough masses to be produced at the LHC, and the physics above this scale is not considered, i.e. the SUSY breaking mechanism. Once a signature has been identified, the objective is to isolate event or object properties where we would expect good discrimination between the SM background and our signal, when the data is observed. This is usually in the form of a set of kinematic selections/cuts required for events to "pass" the requirements of a signal region, and all SUSY searches discussed in this document are based on this method. The analyses are optimised to maximise sensitivity to variations from SM expectations, but if agreement is seen between the SM prediction and the observed data, 95\%CL exclusion limits are set, ruling out scenarios that are incompatible with observation. These limits are usually given as a function of parameters in the model, for example masses of additional particles, couplings, or the production cross-section of the process considered at the LHC. In order to interpret these observations effectively, it is vital that the SM background processes are well modelled and accurately described in the regions of interest. Analyses often incorporate data-driven background estimation methods where possible, and validate the predictions in specifically designed control regions. Many SUSY searches are conducted "blind". This means that the signal regions are designed based solely on theory, and the data must not be observed until everything in the analysis is finalised. This is referred to as "unblinding". For these analyses it is crucial that this testing of the background estimation does not uncover data in the search regions prior to unblinding, so validation of the background estimation

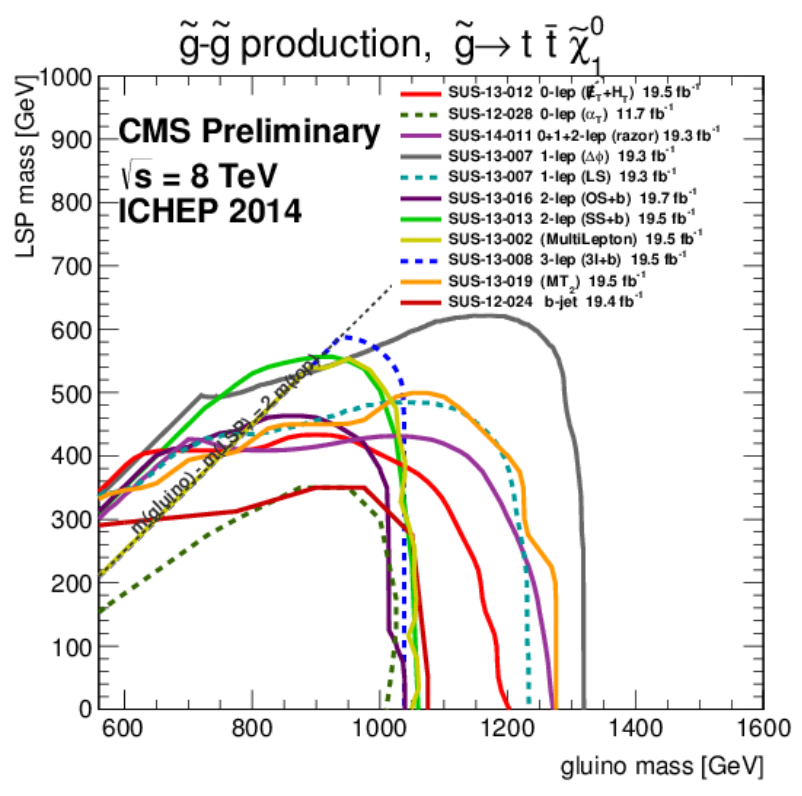

Figure 1. Summary of CMS $95 \%$ CL exclusion limits in strong production scenarios in the $\tilde{\chi}_{1}^{0}$ mass versus $\tilde{g}$ mass plane [9].

must be done in regions with do not have any overlap with the signal regions.

\subsection{Inclusive Strong Production Searches}

Direct production of gluinos and first and second generation squarks have high cross-sections for masses up to around $1 \mathrm{TeV}$. The majority of models consider the decay of these SUSY particles to the lightest sparticle (LSP), which is usually considered to be a neutralino. This will escape the experiment undetected, provided it is stable. In these cases, the LSP provides a good candidate for a weakly interacting dark matter particle. The decay chain leading from the strongly produced sparticles and the LSP will involve the production of a number of SM particles, predominantly hadronic jets and depending on the scenario, sometimes leptons. The escaping LSP provides a source of missing transverse energy, $E_{T}^{\text {miss }}$, a requirement on which is also commonly included in searches.

Recent results from the CMS collaboration include the search for events with three or more leptons [10]. The analysis separates events dependent on the flavour and sign of the contained leptons (of which there must be at least three), and a number of cuts on event properties to discriminate signal from background events. These include the $E_{T}^{m i s s}=-\sum_{i} p_{T}^{i}$, the negative sum of all $i$ visible particle transverse momenta in the event, the jet transverse momentum, $H_{T}=\sum_{j} P_{T}^{j}$, the sum of all $\mathrm{j}$ jet transverse momenta in the event, and the invariant mass of same flavour, opposite charge pairs of leptons, $m_{l l}$. Background is dominated by $\mathrm{WZ}$ and $\mathrm{ZZ}$ processes, which are targeted with tight isolation requirements on the leptons. Results are interpreted in a model with pair-produced gluinos, which each decay via $\tilde{g} \rightarrow t \tilde{t}^{*} \rightarrow t \bar{t} \tilde{\chi}_{1}^{0}$ to produce a final state containing four tops. The exclusion limits set on this 


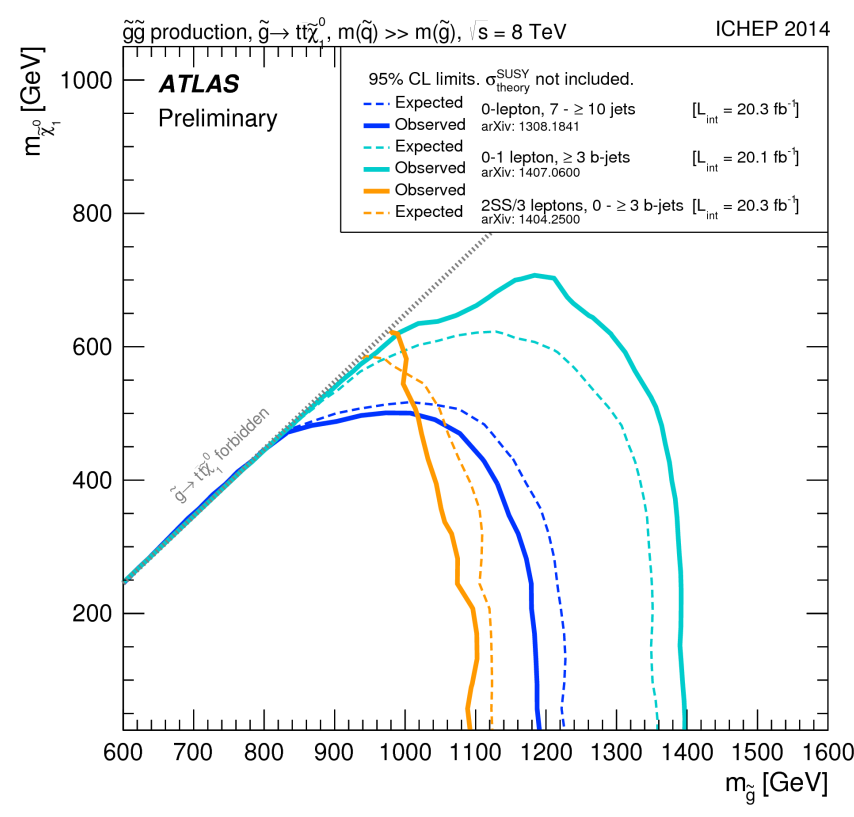

Figure 2. Summary of ATLAS $95 \% \mathrm{CL}$ exclusion limits in strong production scenarios in the $\tilde{\chi}_{1}^{0}$ mass versus $\tilde{g}$ mass plane [8].

grid are included in those shown in Figure 1, which summarises limits from all CMS analyses [9] considering this scenario. In addition, interpretations in Gauge Mediated SUSY Breaking (GMSB) [15], direct sbottom pair production and rare flavour-violating decays of $\mathrm{t} \rightarrow \mathrm{cH}$ are made.

The ATLAS analysis searching for events with three or more b-jets [11] also interprets in the (gluino, neutralino) mass plane. Events with either zero or one leptons, at least three b-jets, and four or more or seven or more jets are selected. In addition, a number of kinematic variables combining the $E_{T}^{\text {miss }}, p_{T}$ of the leptons, and $H_{T}$, and the minimum azimuthal separation between jets, $\delta \phi_{\text {min }}^{\text {jets }}$, are used to define a total of nine signal regions. The background is dominated by the irreducible $t \bar{t}$ process, estimated with a data-driven method based on the matrix method. This employs a system of equations relating the number of b-tagged jets to those which are true, to estimate the probability of jets which are not truly b-jets being b-tagged. Events are weighted by corrections dependent on jet flavour proportions, $p_{T}$ and $\eta$. The prediction is then validated on an inclusive sample of $t \bar{t}$ events. The exclusion limit for gluino production and a neutralino LSP can be seen in Figure 2, along with other recent ATLAS analyses interpreting on this model [8]. The masses of the gluino and neutralino are the parameters which are varied. It can be seen that different analyses (listed in the legends) are useful for different areas of the parameter space, and the maximum effect is gained by considering all final states with sufficient sensitivity.

\subsection{Third Generation Searches}

Direct production of the third generation strong particles, i.e. the stops and sbottoms, are considered separately from

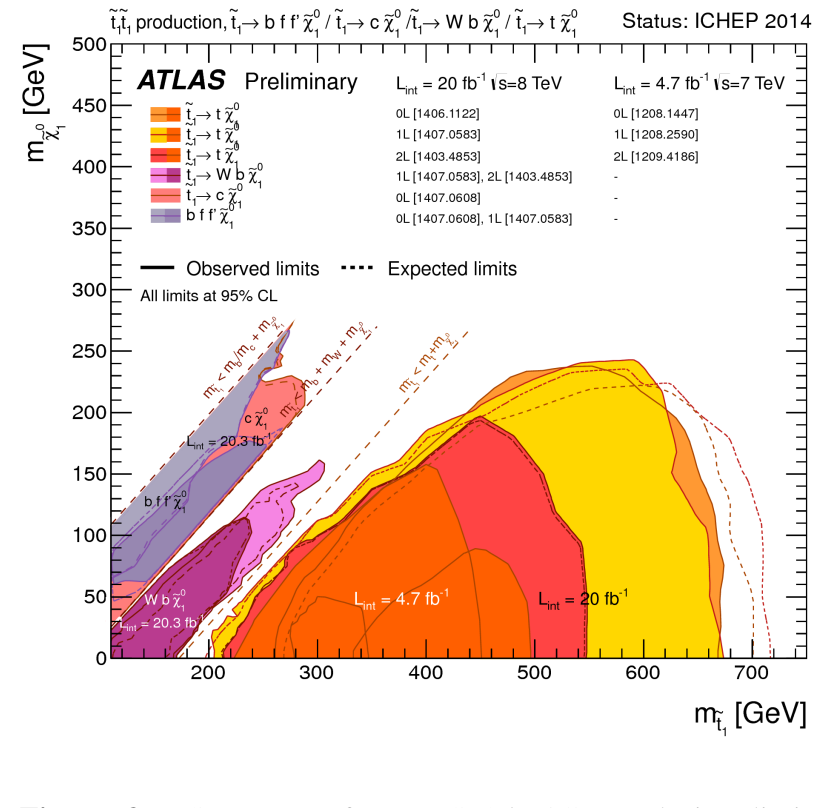

Figure 3. Summary of ATLAS $95 \% \mathrm{CL}$ exclusion limits in strong production scenarios in the $\tilde{\chi}_{1}^{0}$ mass versus $\tilde{t}_{1}$ mass plane [8].

other SUSY searches. They have a more moderate production cross-section than the gluinos and first and second generation squarks, up to masses of aroung $0.5 \mathrm{TeV}$. These decays also produce a large number of jets, possibly leptons, and missing transverse energy, $E_{T}^{\text {miss }}$. The mixing between the mass eigenstates of right- and left-handed stop partners is non-negligible, resulting in two non-degenerate stop states, $\tilde{t}_{1}$ and $\tilde{t}_{2}$, of which $\tilde{t}_{1}$ is the lightest.

The ATLAS search for one lepton and four jets (at least one of which must be a b-jet) [12] targets two, three, and four-body decays of the lightest $\tilde{t}_{1}$ to a $\tilde{\chi}_{1}^{0} \mathrm{LSP}$, with different mass splitting assumptions leading to different SM decay products. Some of these involve a boosted stop, which are targeted by regions with a large required jet separation. Regions are also defined with low $p_{T}$ leptons in order to achieve sensitivity to scenarios which have a low mass stop. Figure 3 summarises ATLAS results from a number of analyses in the $\tilde{\chi}_{1}^{0} \tilde{t}_{1}$ mass parameter space. Different regions of this space correspond to different decay modes of the stop, as these are dependent on the mass different between the $\tilde{t}_{1}$ and $\tilde{\chi}_{1}^{0}$. Lines are included to mark the limits of a given decay, as labelled. The analysis contributes to three regions of the parameter space via these different decay modes, as can be seen by the three separate limits displayed.

The CMS search for one, two, or three or more leptons and one or three b-jets [13] targets a different decay - that of the heavier $\tilde{t}_{2}$ to the lighter $\tilde{t}_{1}$. These decays produce either a $\mathrm{Z}$ or Higgs and a $t \bar{t}$ pair. A total of 96 signal regions are defined, split by the number of leptons and b-jets, and multiple kinematic variables, some of which are "binned", i.e. split into a number of ranges for the parameter value. The most prominent variables are $E_{T}^{\text {miss }}$, transverse lepton 


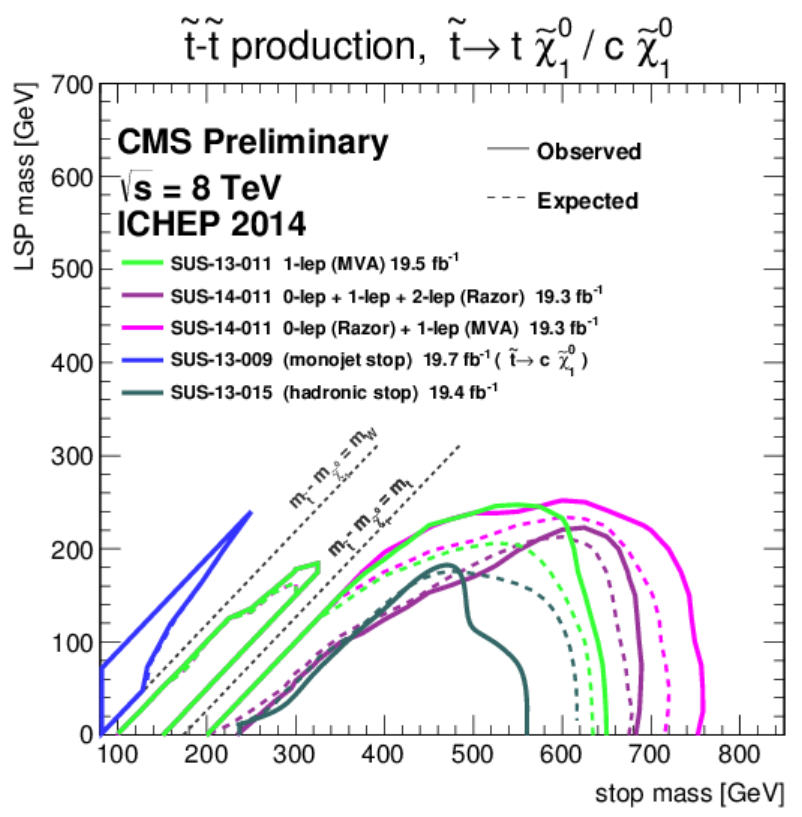

Figure 4. Summary of CMS $95 \%$ CL exclusion limits in strong production scenarios in the $\tilde{\chi}_{1}^{0}$ mass versus $\tilde{t}_{1}$ mass plane [9].

momentum $p_{T}^{l}$, and the transverse mass, $m_{T}$, defined as:

$$
m_{T}=\sqrt{2 E_{T}^{m i s s} p_{T}^{l}\left(1-\cos \left(\Delta \phi\left(E_{T}^{m i s s}, l\right)\right)\right)}
$$

where $\Delta \phi\left(E_{T}^{\text {miss }}, l\right)$ gives the separation in $\phi^{1}$ between the considered lepton and the $E_{T}^{\text {miss }}$. The limits which are set in the $\tilde{t}_{2}, \tilde{t}_{1}$ mass plane indirectly probe the region of $\tilde{\chi}_{1}^{0}, \tilde{t}_{1}$ mass parameter space where the mass difference between the stop and neutralino is approximately that of the top. This can be seen in Figure 4, marked with a dashed line, and is an area where other searches struggle for sensitivity as the signal decay products have very similar kinematic properties to those coming from MC background processes. It can be seen that this causes a region of the paramater space which is not covered by these exclusion limits.

\subsection{Electroweak Production Searches}

Direct production of electroweak SUSY includes production of neutralinos, charginos, and sleptons, and has the lowest cross-sections at the LHC, with sensitivity to detection lost between about 300 and $600 \mathrm{GeV}$ in sparticle mass. However, it could still be the dominant production mode in the case of heavy coloured sparticles, and benefits from final states rich in leptons.

A large range of electroweak production and decay scenarios are covered by the CMS analysis searching for events with one, two, three, or four leptons, and missing

\footnotetext{
${ }^{1}$ A cylindrical co-ordinate system is used to describe both ATLAS and CMS detectors, with the beam pipe aas the $\mathrm{z}$-axis, the $\mathrm{x}$-axis pointing towards the centre of the LHC and the y-axis pointing upwards. All regions of the detector are then described by the angles $\phi$ from the $\mathrm{z}$-axis and $\theta$ around the z-axis.
}

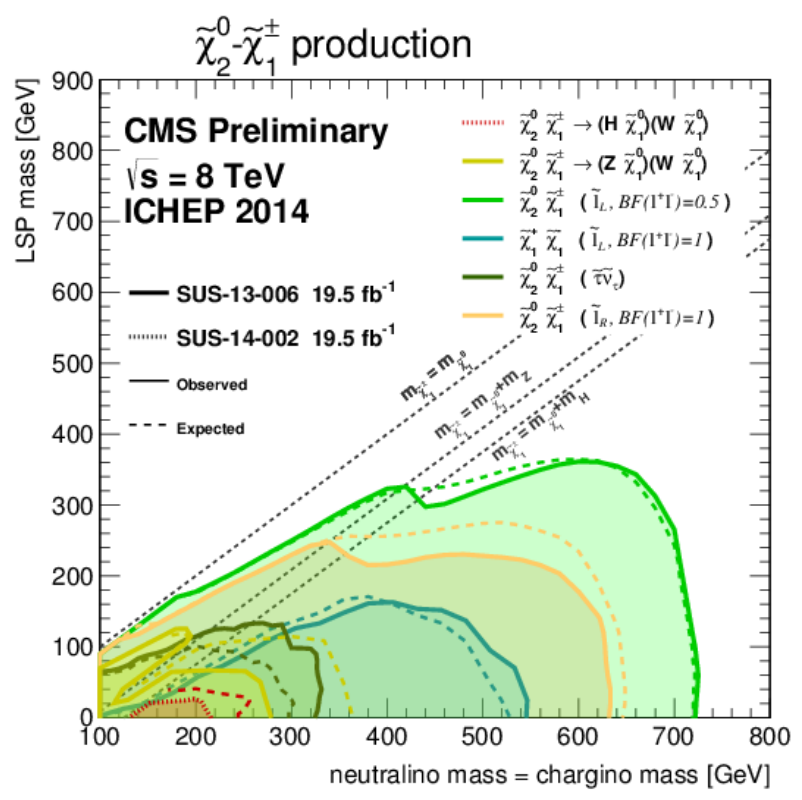

Figure 5. Summary of CMS $95 \%$ CL exclusion limits in electoweak production scenarios in the $\tilde{\chi}_{1}^{ \pm}$mass versus $\tilde{\chi}_{1}^{0}$ mass plane, where a number of decay modes are considered [9].

transverse energy [14]. Regions that require the presence of one lepton target $\tilde{\chi}_{1}^{ \pm} \tilde{\chi}_{2}^{0} \rightarrow \mathrm{WH}$ decays, while two lepton regions are sensitive to direct slepton production and signal producing three leptons in the case that one is lost, three lepton regions target $\tilde{\chi}_{1}^{ \pm} \tilde{\chi}_{2}^{0}$ production with decays through sleptons, or to $\mathrm{ZH}, \mathrm{ZZ}$ or $\mathrm{WH}$, and finally the four lepton region is interpreted in GMSB [15]. Search regions are defined separately for each lepton multiplicity. The four lepton regions are binned in $E_{T}^{\text {miss }}$, and the three lepton regions also used a binned approach for values of lepton invariant mass, $m_{l l}, E_{T}^{\text {miss }}$ and transverse mass $m_{T}$. There are only two regions using two leptons, defined with cuts on the $E_{T}^{\text {miss }}$, and a jet veto for one region. A large number of properties are used to define signal regions which suppress background and enhance signal, including $E_{T}^{\text {miss }}$, lepton transverse mass, $M_{T}^{l}$, and the number of jets. The "mother" particle mass, $M_{T 2}^{b l}$, is also defined and used, constructed as the combined four-momentum of the lepton, b-jets and $E_{T}^{\text {miss }}$ in the event. Exclusion limits for the $\tilde{\chi}_{1}^{ \pm} \tilde{\chi}_{2}^{0}$ for this analysis are displayed in Figure 5 as the solid lines (labelled SUS-13-006) for a variety of decay modes.

ATLAS recently performed an analysis searching for two taus and missing transverse energy [16], with sensitivity to direct stau production, and to $\tilde{\chi}_{1}^{ \pm} \tilde{\chi}_{2}^{0}$ or $\tilde{\chi}_{1}^{ \pm} \tilde{\chi}_{1}^{ \pm}$decays through a stau. Four signal regions are defined with two opposite sign taus, a jet veto, and further cuts on $E_{T}^{\text {miss }}$, combinations of different object transverse masses, or on the effective mass, $m_{e f f}$, defined as:

$$
m_{\text {eff }}=E_{T}^{m i s s}+p_{T}^{\tau_{1}}+p_{T}^{\tau_{2}} .
$$

Background processes are dominated by misidentified jets being reconstructed as taus, and these are modelled from data, using the "ABCD" method. This involves defining 


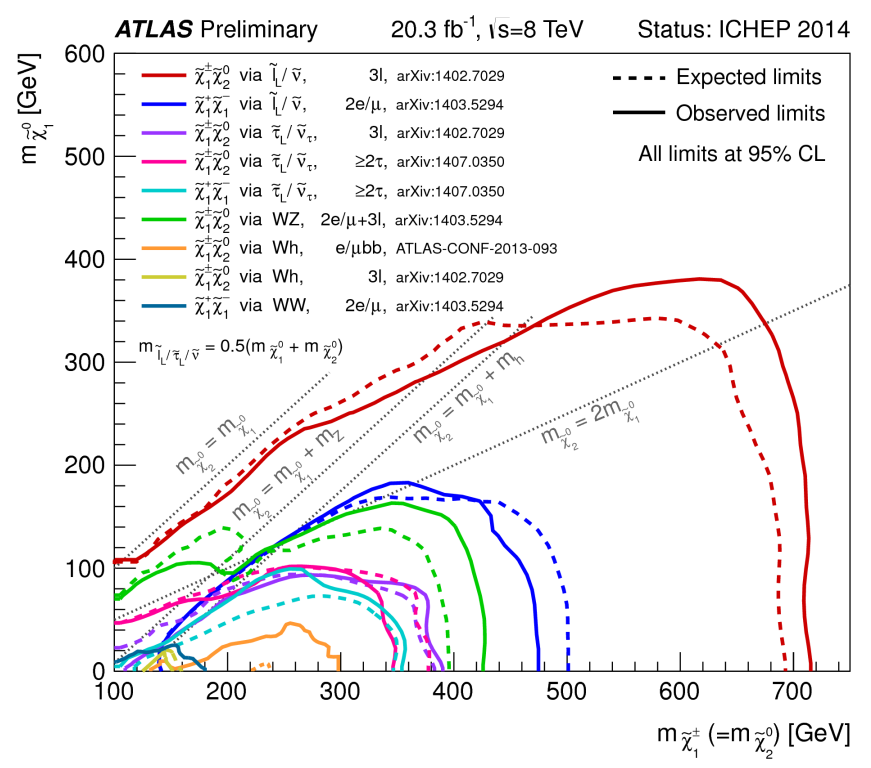

Figure 6. Summary of ATLAS $95 \% \mathrm{CL}$ exclusion limits in electroweak production scenarios in the $\tilde{\chi}_{1}^{ \pm}$mass versus $\tilde{\chi}_{1}^{0}$ mass plane, where a number of decay modes are considered [8].

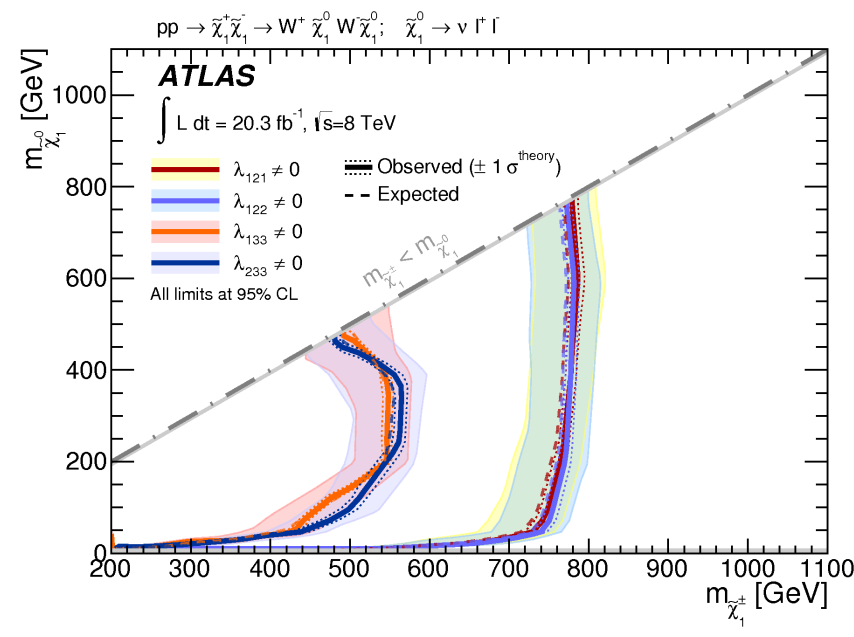

Figure 7. ATLAS four lepton analysis [17] $95 \% \mathrm{CL}$ exclusion limits in RPV scenarios in the $\tilde{\chi}_{1}^{0}$ (LSP) mass versus $\tilde{\chi}_{1}^{ \pm}$(NLSP) mass plane. $\lambda_{121}$ and $\lambda_{122}$ allow decays to electrons and muons only, and $\lambda_{133}$ and $\lambda_{233}$ also include taus.

four regions, the signal region(SR) D and three control regions(CR) A, B and $\mathrm{C}$ using two uncorrelated discriminating variables, for example. The ratio of the number of events in CRs A and B should be equal to the ratio in CR C and SR D. A transfer factor is then calculated and applied to the events in $\mathrm{C}$ to estimate the total in the signal region D. Validation regions are defined to test the performance before unblinding. Exclusion limits for the chargino neutralino decays via staus are shown in Figure 6, complementing the three lepton search targetting the same process by exclusing different regions of the parameter space.

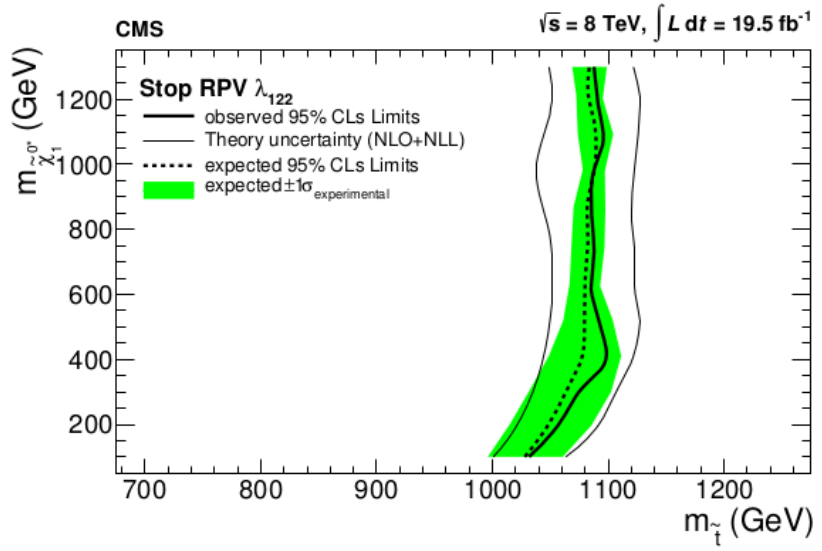

Figure 8. CMS three lepton analysis [18] $95 \% \mathrm{CL}$ exclusion limits in RPV scenario in the $\tilde{\chi}_{1}^{0}$ (LSP) mass versus $\tilde{t}_{1}$ (NLSP) mass plane.

\subsection{R-parity Violating Searches}

ATLAS searches for an R-parity violating(RPV) scenario with lepton number violation in events with four leptons and missing transverse energy [17]. The coupling for the first term in the RPV superpotential given by equation 2 , $\lambda_{i j k} L_{i} L_{j} \bar{E}_{k}$, is set to be non-zero. Couplings with four different variations of indices are considered, $\lambda_{121}$ and $\lambda_{122}$, which result the LSP decaying to electrons and muons, and $\lambda_{133}$ and $\lambda_{233}$, which result in decays which also include taus. Nine signal regions are defined, based on lepton flavour (up to two taus are included), $E_{T}^{\text {miss }}$ and $m_{\text {eff }}$. Limits are set on twenty RPV scenarios, with varying NLSPs and coupling indices. Four of these can be seen in Figure 7, for the case when a chargino is set as the NLSP, for each of the four considered couplings $\left(\lambda_{121}, \lambda_{122}, \lambda_{133}\right.$ and $\left.\lambda_{233}\right)$. Limits are also set in a number of electroweak RPC scenarios.

The CMS analysis searching for events containing three leptons [18] consider the second term of equation 2 , $\lambda_{i j k}^{\prime} L_{i} Q_{j} \bar{D}_{k}$, to be non-zero in addition to the first. The model involves stop pair production and decay to a neutralino LSP, which then decays to leptons. This produces a signature with tops, leptons and neutrinos in the final state. Eight signal regions are defined with different light lepton and tau multiplicities, half including b-jets. All regions are binned in the scalar sum of the $E_{T}^{\text {miss }}$, lepton and jet transverse energies, represented by the variable $S_{T}=E_{T}^{m i s s}+E_{T}^{j e t s}+E_{T}^{l}$. The backgrounds are dominated by real diboson processes, but data-driven estimates are used for the fake components. Limits are set in the stop versus neutralino mass place, as seen in Figure 8.

\section{Exotica Results}

In addition to SUSY, many other BSM theories are considered by both experiments. Due to the number and diversity of the considered models, and the similarity of the signatures many would give at the LHC, these searches will be categorised by the search methods, rather than the model. Some exotica searches are based on similar methods to 


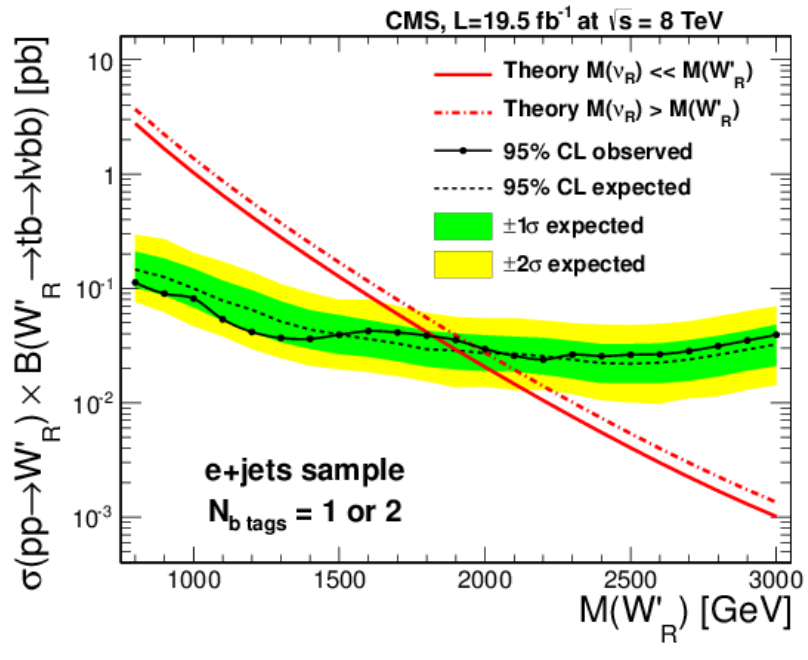

Figure 9. CMS 95\%CL exclusion limit set on the cross-section of a heavy right-handed $\mathrm{W}^{\prime}$ decaying via a semi-leptonic tb pair, as a function of the $\mathrm{W}^{\prime}$ mass [23].

the general SUSY search discussed previously, defining regions in which to compare the number of expected and observed events. There are also some new physics scenarios which distinguished from the background in quite specific and novel ways, for example the long-lived particle searches discussed below, which focus on signatures coming from particles decaying within the detector itself. Background estimation methods tend to vary from analysis to analysis, with some normalising MC simulations, and some employing more sophisticated data-driven techniques.

\subsection{Resonance Searches}

The resonance search method is very versatile in terms of interpretation, but the majority of searches make cuts in order to select objects as similar to those which would be produced by a given signal as possible, whilst suppressing background processes. An example of signal which searches have optimised for is the presence of heavy spin$1 \mathrm{~W}^{\prime}$ bosons. These can result from many BSM theories, including Grand Unified Theories [19], Little Higgs [20], Technicolor [21] and Extra Dimensions [22].

CMS conducted a resonance search based on a righthanded $\mathrm{W}^{\prime}$ decaying to a top quark and bottom quark [23], where the subsequent top decay is semi-leptonic. In order to select the best candidate $\mathrm{W}^{\prime}$ decay products, events are selected with a high $p_{T}$ electron or muon, $E_{T}^{\text {miss }}>20 \mathrm{GeV}$, and at least 2 jets, one of which must be b-tagged. The invariant mass is constructed for the group of these objects in each event which best resembles the expected signature of a $\mathrm{W}^{\prime}$ decaying to produce a top and bottom quark, referred to collectively as tb candidates. The invariant mass distribution for all events is then compared to the SM estimated background, and a resonance above $1500 \mathrm{GeV}$ would correspond to a signal. 95\% CL limits are set on the $\mathrm{W}^{\prime}$ production cross-section, as a function of $\mathrm{W}^{\prime}$ mass, as seen in Figure 9.

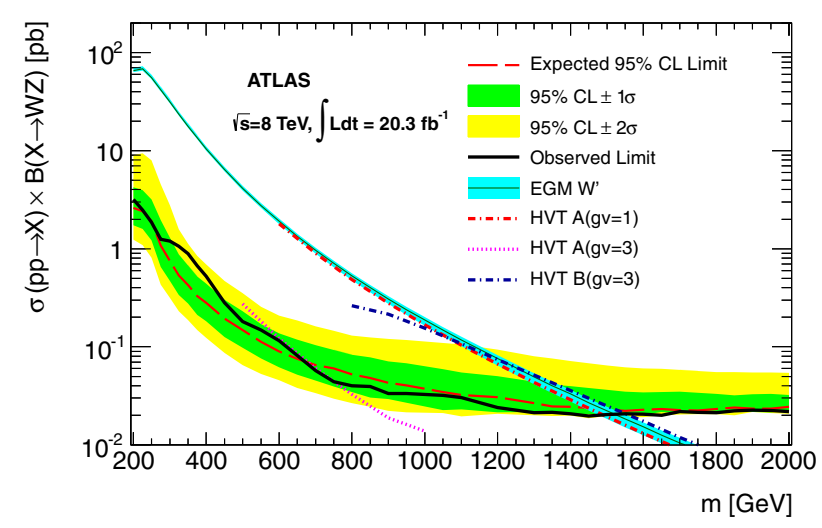

Figure 10. ATLAS 95\%CL exclusion limit on the cross-section of a heavy $\mathrm{W}^{\prime}$ decaying via a fully leptonic $\mathrm{WZ}$ pair, as a function of the $\mathrm{W}^{\prime}$ mass [24].

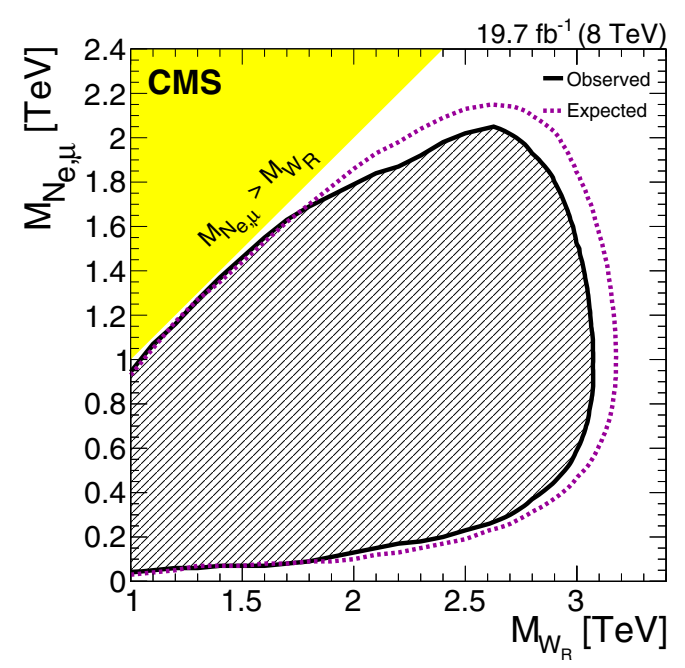

Figure 11. CMS 95\%CL exclusion limit in the heavy neutrino $N_{l}$ versus $W_{R}$ mass plane. $W_{R}$ is required to always be heavier due to the assumed decay mode [25].

A complementary search for $\mathrm{W}^{\prime}$ decaying to a $\mathrm{WZ}$ pair, which then decays via the fully leptonic channel [24] was carried out by the ATLAS experiment. Similarly to the CMS search, events containing potential WZ decay products are identified, before using these objects to reconstruct a candidate WZ. As the fully leptonic WZ decay mode is considered, three charged leptons are required, two of which need to be an opposite sign, same flavour pair with an invariant mass within $10 \mathrm{GeV}$ of the $\mathrm{Z}$ boson pole mass (the pair closest in mass is selected if there are multiple choices). Finally a request is made on the rapidity difference between this lepton pair and the remaining lepton, which should have originated from the $\mathrm{W}$ boson. The invariant mass of the WZ candidates are compared to SM background, where the considered signal can produce resonances from around $600 \mathrm{GeV}$. Limits are set on the $\mathrm{W}^{\prime}$ cross-section as a function of mass, shown in Figure 10, as well as on couplings for a heavy vector triplet scenario, a benchmark model for heavy vector bosons. 


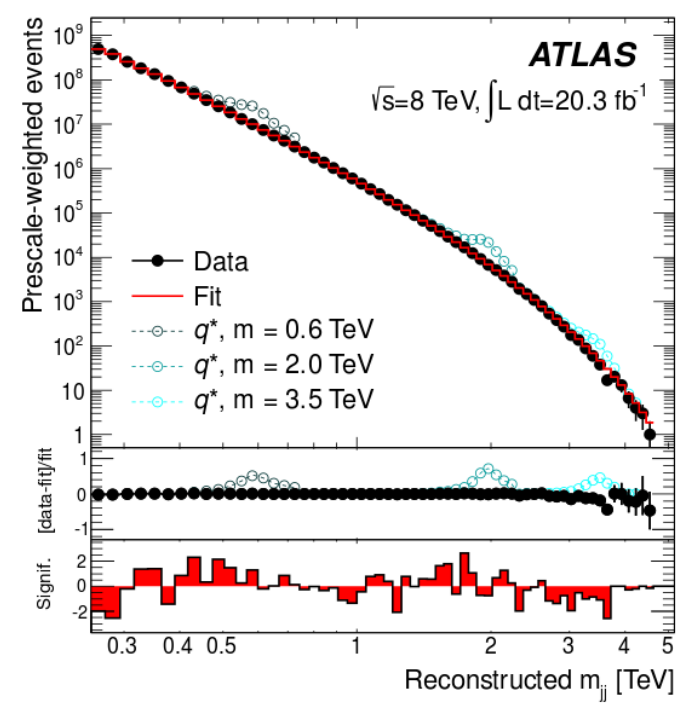

Figure 12. Invariant mass of dijet pairs for SM background compared to three excited quark signals, and the observed data, which is in agreement with background predictions [26].

CMS also conducts a search for a right-handed heavy $\mathrm{W}$ boson, $\mathrm{W}_{R}$, in conjunction with heavy neutrinos, both of which originate from a left-right (LR) symmetric extension of the Standard Model [25]. The decay chain is assumed as $W_{R} \rightarrow l_{1} N_{l} \rightarrow l_{1} l_{2} W_{R}^{*} \rightarrow l_{1} l_{2} q \bar{q}$, (where $N_{l}$ is a heavy neutrino, and $W_{R}^{*}$ is a virtual $W_{R}$ ) leading to a final state with leptons and jets. The invariant mass of the combination of the two highest $p_{T}$ leptons and two highest $p_{T}$ jets is taken, where events are split into those containing either muon or electron pairs. The background is estimated using the invariant mass distribution of $e \mu$ pairs, which it is shown should be analagous to pairs of just electrons or muons. Expected mass resonances are of the order of a few $\mathrm{TeV}$, and as agreement with SM background is observed, limits are set in the heavy neutrino, $\mathrm{W}_{R}$ mass plane, as seen in Figure 11.

Finally we present results from a dijet mass resonance search [26] which constructs the invariant mass of the two highest $p_{T}$ jets in each event, and interprets in a total of seven different models which could produce resonances in this distribution. Some of these include quantum black holes, either lepto-phobic or lepto-phillic excited W decays to quark pairs, and excited quarks, the distribution including expected resonances for which can be seen in Figure 12. The background estimation is data-driven, using a functional form which allows for small variation within the background but not localised excesses which could correspond to a BSM signal. Agreement is seen between SM background predictions and the observed data. Many exclusion limits are set on the cross-sections of BSM scenarios considered, as well as on the masses of many proposed heavy BSM particles, e.g. $4.09 \mathrm{TeV}$ on excited quarks and around 5.8 TeV on two kinds of black holes. The sensitivity is not limited to the scenarios considered though, and any signal producing two high $p_{T}$ jets could make use of this analysis, dependent on cross-section.

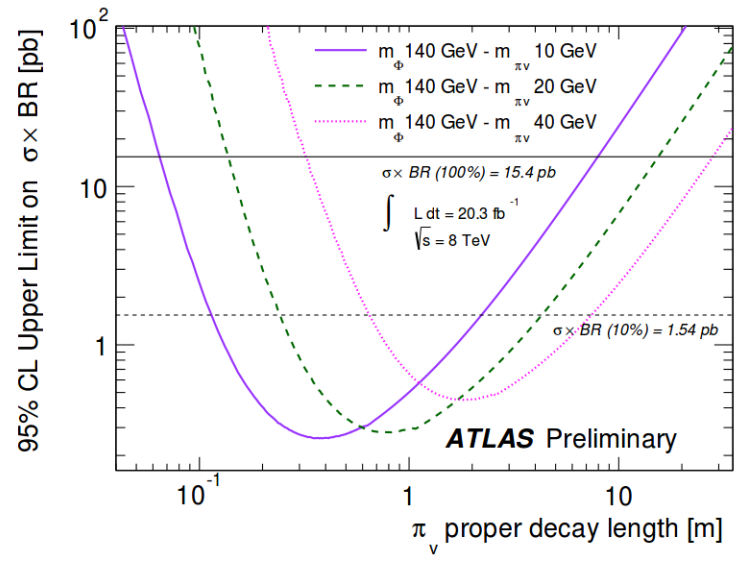

Figure 13. $95 \% \mathrm{CL}$ upper limits on the cross-section of Hidden Valley pions with varying masses, as a function of their decay length [27].

\subsection{Long-lived Particle Searches}

Scenarios which result in long-lived particles require alternative search techniques which focus on decays which are displaced within the detector.

A recent example from ATLAS [27] considers longlived neutral particles resulting from a "Hidden Valley" (HV) [28] model, where a hidden sector is coupled to the Standard Model sector via a heavy scalar "communicator" particle. In this instance, there exists a triplet of HV particles, referred to as pions, which are pair-produced at the LHC. Jets are selected which do not have any associated "good" tracks from the inner tracking detector, where "good" requires a certain number of hits in the various pixel and silicon layers. This is what we would expect from jets originating from within the detector itself, produced after a massive particle has passed through the inner tracking detector. This gives a very good discrimination against the SM background, and additional timing cuts are made on the jet energy deposition, to ensure that they correspond to a pp collision in the centre of the detector. Two jets satisfying these cuts are selected, and the yields are compared to the prediction for Standard Model multijet and cosmic predicted background processes. These are in agreement within uncertainties, allowing 95\%CL upper limits to be placed on the cross-section as a function of the decay length of the HV pions, which can be seen in Figure 13.

R-parity violating supersymmetry scenarios can also lead to long-lived particles if the coupling is weak enough, giving a displaced decay of the metastable LSP. This interpretation is considered by a CMS analysis focussing on leptons with large impact parameters with respect to the beam line location [29], where the LSP is a stop which is pair-produced, giving an electron-muon pair in the displaced decay. The electron and muon are required to be well seperated within the detector, and the impact parameter requirement for the electron and muon is varied in different bins to maintain sensitivity to a number of scenarios. The majority of background is estimated from MC, with correction scale factors applied, except for the heavy- 


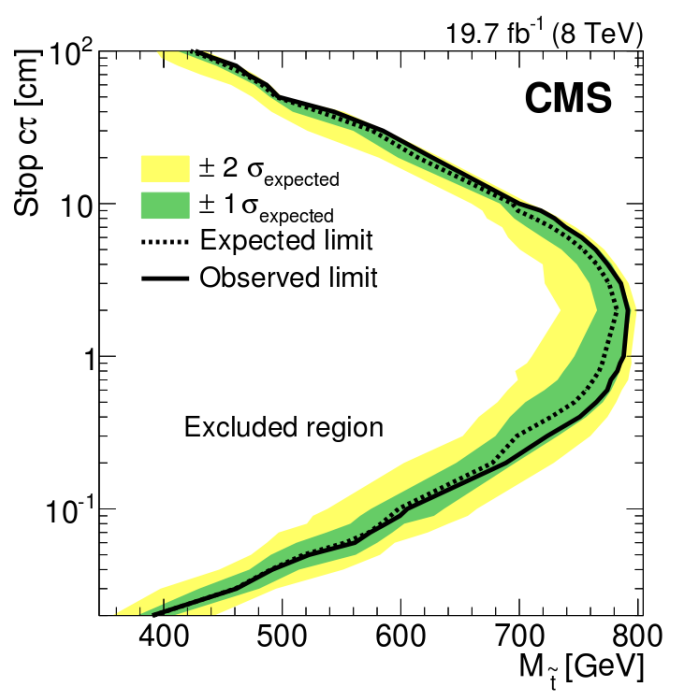

Figure 14. CMS displaced dilepton analysis $95 \%$ CL exclusion limits on the stop LSP as a function of it's lifetime, for a weakly R-parity violating supersymmetry scenario [29].

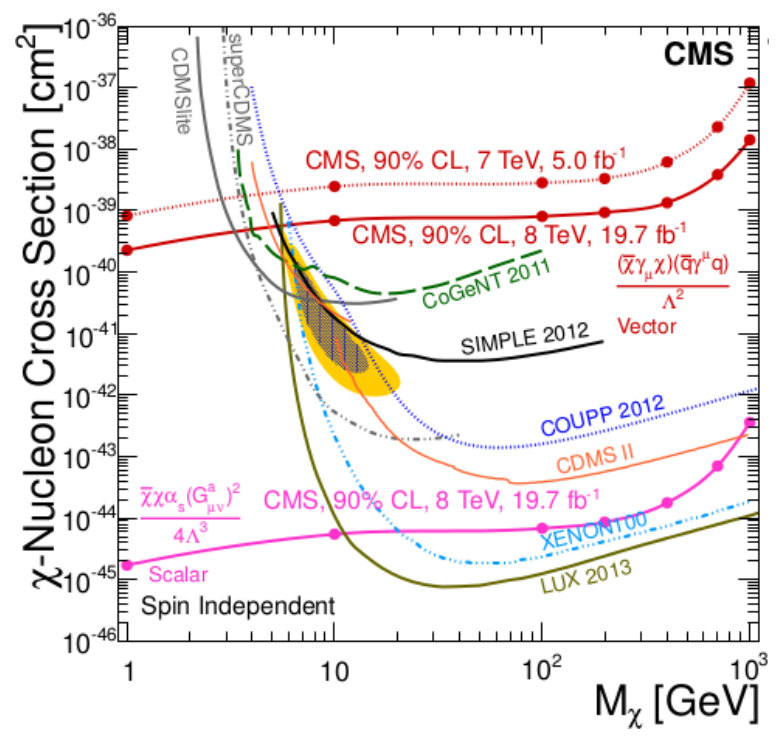

Figure 15. CMS monojet analysis $95 \% \mathrm{CL}$ exclusion limit on dark matter nucleon cross-section as a function of ucleon mass, compared to a number of dark matter experiment limits [30].

flavour QCD sample, which is taken from data. Regions with slightly different requests to the signal regions are defined, in order to calculate the ratios of events in each region, and transfer this to the signal region. The results for the $95 \%$ CL limit on the stop mass as a function of its lifetime are shown in Figure 14.

\subsection{Other Searches}

Recent exotica searches of interest which do not fit into the two previous categories are also presented for each of the experiments.

CMS has carried out a search which is interpreted in a number of different models, using a signature of monojet events [30]. One or two jets are selected, requir-

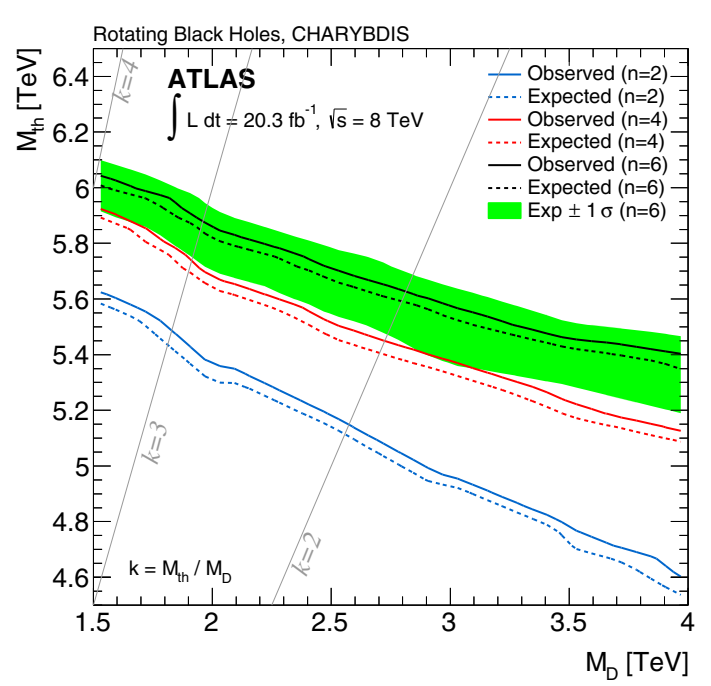

Figure 16. ATLAS one lepton plus jets analysis 95\%CL limits on rotating black holes in the $M_{t h}$ versus $M_{D}$ mass plane, for ADD extra-dimensional models with $\mathrm{n}=2,4$ and 6 [34].

ing that they are within a given separation in the second instance, in order to suppress background from QCD. Events with more than two jets or any leptons are vetoed. Seven regions are then defined with varying $E_{T}^{\text {miss }}$ requirements, and the number of remaining data events in each is compared with the Standard Model background prediction. Observations are all consistent with the estimated background within uncertainties, and so limits are set on a number of BSM models which can result in the presence of dark matter. These include contact interactions[31], ADD extra dimensions (Arkani-Hamed, Dimopolous, Dvali) [32] and unparticle production[33]. Limits on the dark matter nucleon cross-section as a function of nucleon mass are shown in Figure 15, in comparison with existing limits from other experiments searching for dark matter.

The ATLAS one lepton plus jets analysis [34] selects events with three or more high $p_{T}$ leptons or jets, where at least one must be a lepton, and uses the sum of their $p_{T}$ to define binned signal regions. $\Sigma p_{T}$ must be at least $2 \mathrm{TeV}$. This provides a good discrimination between background and signals for ADD extra dimensional models where a black hole is produced and decays. As agreement between data and prediction is seen, limits are set in the mass plane of parameters of the black holes, $M_{t h}$, the threshold mass, and $M_{D}$, the scale of quantum gravity. Limits are set for a number of different ADD models and scenarios with both rotating and non-rotating black holes. The result for rotating black holes with one of the two considered simulations can be seen in Figure 16.

\section{Outlook}

Run 1 at the LHC may not have yielded any discoveries for theories of BSM physics, but it has allowed us to greatly constrain a wide range of scenarios, and narrow 
down the best places to continue looking during Run 2 . 95\% CL limits have been placed on supersymmetry models with strong production, electroweak production and cases where R-parity is violated, even in cases where this is weak and leads to a long-lived LSP. Constraints have also been placed on models resulting in heavy vector bosons, heavy pions, excited quarks, black holes, and dark matter. Many analyses could also have sensitivity to models not yet considered, and could place bounds on new theories.

In 2015, the LHC will restart at a centre-of-mass energy of $13 \mathrm{TeV}$ - almost double that used for Run 1. This will affect the cross-sections of all physics processes, both SM and BSM. The ratio of cross-sections at the new energy compared to the old is predicted at around 2-2.5 for Higgs processes, and around 4 or 5 for $t \bar{t}$ processes. By comparison, stop pair production expects to see a boost of around a factor of 10, and gluino production even higher, up to 2700 times the cross-section in Run 1. SUSY processes are not the only promising BSM signatures for the new start-up, $Z^{\prime}$, excited quarks and quantum black holes are predicted a factor of 13,87 , and 12000 increase in cross-section. This will greatly improve the discovery potential of many BSM searches, giving a very optimistic outlook for the LHC Run 2.

\section{References}

[1] ATLAS Collaboration, Physics Letters B, 716, 1-29 (2012)

[2] CMS Collaboration, Physics Letters B, 716, 30-61 (2012)

[3] L.Evans and P.Bryant, JINST, 3, S08001 (2008)

[4] ATLAS Collaboration, JINST, 3, S08003 (2008)

[5] CMS Collaboration, JINST, 3, S08004 (2008)

[6] S. Wienberg, Physics Review D, 13, 974 (1976)

[7] Stephen P. Martin, arXiv:hep-ph/9709356 (2011)

[8] ATLAS

Collaboration, https://atlas.web.cern.ch/Atlas/GROUPS/PHYSICS/ CombinedSummaryPlots/SUSY/ (2014)

[9] CMS

Collaboration, https://twiki.cern.ch/twiki/bin/view/CMSPublic/ SUSYSMSSummaryplots8TeV (2014)
[10] CMS Collaboration, Physical Review D, 90, 032996 (2014)

[11] ATLAS Collaboration, JHEP, 10, 24 (2014)

[12] ATLAS Collaboration, arXiv:1407.0583, (2014)

[13] CMS Collaboration, Physics Letters B, 736, 371-397 (2014)

[14] CMS Collaboration, EPJC, 74, 3036 (2014)

[15] Joshua T.Ruderman, David Shih, JHEP, 08, 159 (2012)

[16] ATLAS Collaboration, JHEP, 10, 096 (2014)

[17] ATLAS Collaboration, Physical Review D, 90, 052001 (2014)

[18] CMS Collaboration, Physical Review Letters, 111, 221801 (2014)

[19] H.Georgi and S.Glashow, Physics Review Letters, 32, 438-441 (1974)

[20] M.Perelstein, Prog. Part. Nucl. Phys, 58, 247-291 (2007)

[21] E.Malkawi, T.M.Tait and C.P.Yuan, Physics Letters B, 385, 301 (1996)

[22] G.Burdman, B.A. Dobrescu and E.Ponton, Physics Review D, 74, 075008 (2006)

[23] CMS Collaboration, JHEP, 05, 108 (2014)

[24] ATLAS Collaboration, Physics Letters B, 737, 223243 (2014)

[25] CMS Collaboration, arXiv:1407.3683 (2014)

[26] ATLAS Collaboration, arXiv:1407.1376 (2014)

[27] ATLAS Collaboration, ATLAS-CONF-2014-041 (2014)

[28] M.J.Strassler and K.M.Zurek, Physics Letters B, 651, 374-379 (2007)

[29] CMS Collaboration, arXiv:1409.4789 (2014)

[30] CMS Collaboration, arXiv:1408.3583 (2014)

[31] M.Beltran et al., JHEP, 09, 037 (2010)

[32] N.Arkani-HAmed, S.Dimopoulos, G.Dvali, Physics Letters B, 429, 263 (1998)

[33] H.Georgi, Physics Review Letters, 98, 221601 (2007)

[34] ATLAS Collaboration, JHEP, 08, 103 (2014) 